\title{
Acupuncture for diabetic peripheral neuropathy: study protocol for a randomized, placebo-controlled trial
}

Haiping Deng ${ }^{1,2 \dagger}$, Yu Shu ${ }^{1 \dagger}$, Peiran Lv' ${ }^{1}$ Ling Zhao ${ }^{1}$, Ke Cheng ${ }^{1,2}$, Tingting Zhang ${ }^{1}$, Yi Song ${ }^{3}$, Hua Yang ${ }^{3}$, Hong Tang ${ }^{3}$, Jian Pei ${ }^{3^{*+}}$ and Xueyong Shen ${ }^{1 *+}$ (D)

\begin{abstract}
Background: Diabetic peripheral neuropathy (DPN) is the most common chronic complication of diabetes mellitus that has a considerable impact on quality of life, but there are few effective therapeutic strategies. The aim of this trial is to determine the efficacy and safety of manual acupuncture (MA) versus sham acupuncture (SA) for DPN.

Methods/design: This is a study protocol for a randomized, placebo-controlled clinical trial. A total of 118 patients with DPN will be recruited and randomly assigned in a 1:1 ratio to either the MA group or SA group. All patients will receive 24 sessions over 12 weeks. Participants will complete the trial by visiting the research center at month 6 for a follow-up assessment. The primary outcome is peroneal motor nerve conduction velocity (peroneal MNCV) at week 12 compared with baseline. Secondary outcomes include peroneal motor nerve action potential amplitude (peroneal MNAP) and latent period (peroneal MNLP), sural sensory nerve conduction velocity (sural SNCV), action potential amplitude (sural SNAP) and latent period (sural SNLP), fasting plasma glucose (FPG), 2-h postprandial blood glucose (2hPG), glycated hemoglobin (HbAlc) at week 12 compared with baseline, Michigan Neuropathy Screening Instrument (MNSI) score and Diabetes Specific Quality of Life scale (DSQL) at week 12 and month 6 compared with baseline. Safety will be assessed during the whole trial. Masking effectiveness will be assessed by patients.
\end{abstract}

Discussion: This trial may provide high-quality evidence for evaluating the efficacy and safety of MA treatment for DPN compared with SA treatment. Results of this study will be published in peer-reviewed journals.

Trial registration: Chinese Clinical Trials Registry ChiCTR1800020444. First registered on 29 December 2018, retrospectively registered, http://www.chictr.org.cn/showproj.aspx?Proj=31063.

Keywords: Acupuncture, Diabetic peripheral neuropathy, Randomized controlled trial, Study protocol

\footnotetext{
*Correspondence: peijian99@yahoo.com; sxy1@shutcm.edu.cn

${ }^{+}$Haiping Deng, Yu Shu, Jian Pei and Xueyong Shen contributed equally to this work.

${ }^{3}$ Long-Hua Hospital affiliated to Shanghai University of Traditional Chinese Medicine, Xuhui District, Shanghai, China

${ }^{1}$ School of Acupuncture-Moxibustion and Tuina, Shanghai University of Traditional Chinese Medicine, Pudong District, Shanghai, China

Full list of author information is available at the end of the article
}

(C) The Author(s). 2020 Open Access This article is licensed under a Creative Commons Attribution 4.0 International License, which permits use, sharing, adaptation, distribution and reproduction in any medium or format, as long as you give appropriate credit to the original author(s) and the source, provide a link to the Creative Commons licence, and indicate if changes were made. The images or other third party material in this article are included in the article's Creative Commons licence, unless indicated otherwise in a credit line to the material. If material is not included in the article's Creative Commons licence and your intended use is not permitted by statutory regulation or exceeds the permitted use, you will need to obtain permission directly from the copyright holder. To view a copy of this licence, visit http://creativecommons.org/licenses/by/4.0/ The Creative Commons Public Domain Dedication waiver (http://creativecommons.org/publicdomain/zero/1.0/) applies to the data made available in this article, unless otherwise stated in a credit line to the data. 


\section{Background}

Diabetic peripheral neuropathy (DPN) is the most common cause of neuropathy worldwide, and its prevalence increases with the duration of diabetes [1]. Approximately $50 \%$ of patients with diabetes will develop peripheral neuropathy [1-3]. DPN is symmetric and predominantly sensory, starting distally and gradually spreading proximally in a glove-and-stocking distribution [1]. DPN is associated with the development of foot problems such as foot ulcers and is one of the leading causes of amputations. The high-frequency underreport and undertreatment of DPN can lead to an increased risk for morbidity and mortality and decreased quality of life $[1,2]$. Once symptoms appear, there are few effective therapeutic strategies [4]. While neuropathic pain and paresthesia can be palliated by anti-convulsant drugs, tricyclic antidepressant drugs, or serotonin-noradrenalin reuptake inhibitors [5], pharmacological management of decreased sensation is generally ineffective. Therefore, more and more patients turn to seek nonpharmacological treatments. Multiple complementary and alternative medicine therapies such as acupuncture and yoga have shown efficacy in the treatment of painful peripheral neuropathy [6]. A pilot RCT has demonstrated practicality and feasibility of acupuncture as an additional well-tolerated treatment without appreciable side effects for people with DPN [7]. Acupuncture may effectively ameliorate selected DPN symptoms, such as aching pain, burning pain, prickling sensation, numbness, and allodynia in American Indian patients [8]. Reviews concerning acupuncture for peripheral neuropathy (PN) have found that, despite the majority of studies reporting positive results, a reliable statement of effectiveness is not possible due to methodological limitations such as unsuitable control group and blinding [9-11]. Also, inconsistency of outcome measures and lack of use of global neuropathyspecific patient-reported outcome measures have confounded results. Overall, the quality of acupuncture intervention delivered in individual studies was low. Appropriately powered RCTs with suitable control groups, validated and proper outcome measures for DPN patients, and more accurate reporting of acupuncture interventions, including point selection rationales, point locations, and practitioner qualifications, are needed. Therefore, we have designed a randomized, sham-controlled, participant-blinded and assessor-blinded study with a 6month observation period to explore its effectiveness and safety.

\section{Methods}

\section{Study design}

This randomized, participant-blind, sham-controlled trial will be conducted at Long-Hua Hospital affiliated to Shanghai University of Traditional Chinese Medicine in
China. The protocol for this trial is reported based on the Standard Protocol Items: Recommendations for Interventional Trials (SPIRIT) 2013 Checklist: defining standard protocol items for clinical trials (Additional file 1). The study has been approved by the Ethics Committee of Long-Hua Hospital (Ethical approval number: 2016LCSY028) and registered on the Chinese Clinical Trial Registry (Chictr) platform since 29 December 2018 (Registration number: ChiCTR1800020444). A flow diagram of the trial is shown in Fig. 1.

\section{Patient recruitment}

Patients meet the American Diabetes Association (ADA) 2012 diagnostic criteria for DPN will be recruited from the endocrinology departments of Long-Hua Hospital affiliated to Shanghai University of Traditional Chinese Medicine in Shanghai, China. Recruitment posters will be posted in the hospital and uploaded to social media (WeChat) from October 2016 to December 2021. All patients will be required to provide written informed consent (Additional file 2) before randomization.

\section{Inclusion criteria}

Participants who meet all the following requirements will be allowed for enrollment:

1. Aged $18-75$ years (either sex)

2. Patients who meet diagnosis of DPN defined by ADA in 2005 [12]

a. DPN is defined as the presence of symptoms and/or signs of peripheral nerve dysfunction in people with diabetes, after the exclusion of other causes

b. NP is defined as altered sensitivity to pressure and (1) altered sensitivity to pain or (2) altered sensitivity to vibration or (3) achilles reflex [13]

3. Symmetric and predominantly sensory, starting from the lower limbs distally and gradually spreading proximally in a glove-and-stocking distribution

4. Ability to understand study procedures and willingness to comply with them for the entire period of study

5. Written informed consent

\section{Exclusion criteria}

Participants meeting any of the following criteria will be excluded:

1. PN caused by conditions other than diabetes (e.g., alcohol abuse, chemotherapy, hereditary causes, chronic inflammatory, or idiopathic PN)

2. Psychiatric illnesses other than mild depression 


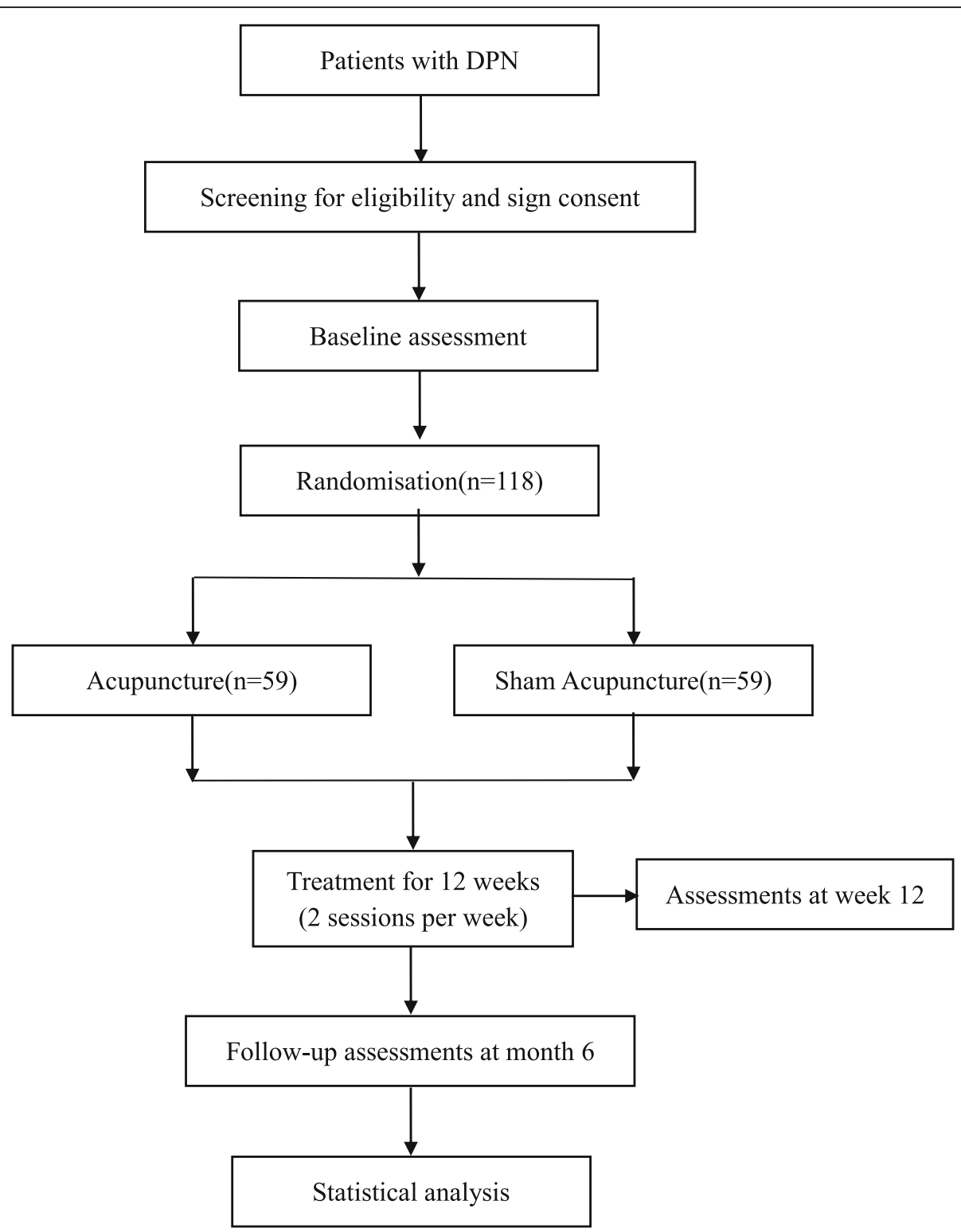

Fig. 1 Flow diagram

3. Severe or unstable cardiovascular, liver, kidney, respiratory, or hematological disorders

4. Received acupuncture treatment in the last 3 months

5. Pregnant or lactating women

6. Research unit personnel directly related to the study and their immediate family members

7. Incapable of giving informed consent or following the study instructions due to language disturbances, serious cognitive deficits, or lack of time

8. Currently participating in other clinical trials

\section{Randomization and allocation concealment}

All eligible patients will be randomly assigned to manual acupuncture (MA) group or sham acupuncture (SA) group in a 1:1 ratio. The blocked randomization sequence will be computer-generated (Excel) by an independent statistician, who will not be involved in implementation and statistical analysis of the trial. The allocation will be placed inside sequentially numbered sealed opaque envelopes which will be opened only after enrollment. The treatment allocation will be revealed to acupuncturists out of patients' sight to ensure blinding. To reduce the risk of observer bias, the acupuncture practitioners will be discouraged from discussing treatments or previous results with patients. The random allocation sequence and sealed opaque envelopes will be kept separately by two specific researchers. The clinical research coordinator will be responsible for enrolling patients, obtaining informed consent, and requesting randomization. 


\section{Masking}

Due to the nature of acupuncture, masking of acupuncturists is quite difficult to achieve. Patients, outcome assessors, and statisticians who perform the statistical analyses will be blinded to group assignment. The treatments subjects received will be not revealed until the statistical analysis is completed.

\section{Interventions}

Treatment will be performed by licensed acupuncturists who have at least 5 years of experience in acupuncture. All the acupuncturists will be trained how to locate acupoints, puncture, and manipulate needles before trials. A placebo device $[14,15]$ will be applied in both groups for better implementation of blindness. Both MA and SA treatments will consist of 24 sessions over 12 weeks ( $40 \mathrm{~min}$ per session, two sessions per week). Acupuncture will be discontinued if patients suffer from any serious adverse events (AEs).

\section{Manual acupuncture}

Patients allocated to the MA group will receive treatment with needles being inserted at the prespecified acupuncture points through the placebo device. Obligatory and additional acupoints adopted in this protocol are developed from clinical experiences of acupuncture experts. The obligatory acupoints include Zhongwan (CV12), bilateral Weiwanxiashu (EX-B3), bilateral Ganshu (BL18), bilateral Pishu (BL20), bilateral Shenshu (BL23), bilateral Zusanli (ST36), bilateral Yanglingquan (GB34), bilateral Sanyinjiao (SP6), bilateral Taixi (KI3), and Bafeng (EX-LE10). Additional acupoints Baxie (EXUE9) will be added when patients' symptoms appear not only in the lower limbs but also in the upper limbs. All acupoints are localized according to the WHO Standard Acupuncture Locations and exhibited in Table 1 and Fig. 2. After skin disinfection, disposable, stainless steel acupuncture needles $(0.25 \times 25 \mathrm{~mm}$ or $0.25 \times 40$ $\mathrm{mm}$, provided by Wuxi Jiajian Medical Instrument Co. Ltd., China) will be inserted into the skin of acupoint (approximately $10-20 \mathrm{~mm}$ depth), and then manipulations of twirling, lifting, and thrusting will be performed on all needles for at least $10 \mathrm{~s}$ to reach De qi (a compositional sensation including soreness, numbness, distention, and heaviness), which is believed to be an essential component for acupuncture efficacy. Firstly, CV12, ST36, GB34, SP6, KI3, EXLE10, and EX-UE9 will be punctured when patients lie supine. Then, EX-B3, BL18, BL20, and BL23 will be punctured when patients lie prone. Needles will be retained in these acupoints for $20 \mathrm{~min}$.

Table 1 Locations and manipulations of acupoints

\begin{tabular}{|c|c|c|c|}
\hline & Acupoint & Location & Manipulation \\
\hline \multirow[t]{10}{*}{ Obligatory acupoints } & $\begin{array}{l}\text { Zhongwan } \\
(C V 12)\end{array}$ & On the anterior midline, 4 cun $^{a}$ above the umbilicus & $\begin{array}{l}\text { Puncture perpendicularly to a } \\
\text { depth of } 1.0-1.2 \text { cun }\end{array}$ \\
\hline & $\begin{array}{l}\text { Weiwanxiashu } \\
(E X-B 3)\end{array}$ & $\begin{array}{l}\text { at the same level as the inferior border of the spinous process of the 8th } \\
\text { thoracic vertebra (T8), } 1.5 \text { cun lateral to the posterior median line }\end{array}$ & $\begin{array}{l}\text { Puncture obliquely and } \\
\text { medially to a depth of } 0.5- \\
0.8 \text { cun }\end{array}$ \\
\hline & Ganshu (BL18) & $\begin{array}{l}\text { at the same level as the inferior border of the spinous process of the 9th } \\
\text { thoracic vertebra (T9), } 1.5 \text { cun lateral to the posterior median line }\end{array}$ & $\begin{array}{l}\text { Puncture obliquely and } \\
\text { medially to a depth of } 0.5- \\
0.8 \text { cun }\end{array}$ \\
\hline & Pishu $(B L 20)$ & $\begin{array}{l}\text { at the same level as the inferior border of the spinous process of the } 11 \text { th } \\
\text { thoracic vertebra }(T 11), 1.5 \text { cun lateral to the posterior median line }\end{array}$ & $\begin{array}{l}\text { Puncture obliquely and } \\
\text { medially to a depth of } 0.5- \\
0.8 \text { cun }\end{array}$ \\
\hline & $\begin{array}{l}\text { Shenshu } \\
(B L 23)\end{array}$ & $\begin{array}{l}\text { at the same level as the inferior border of the spinous process of the } 2 \text { nd } \\
\text { lumbar vertebra (L2), } 1.5 \text { cun lateral to the posterior median line }\end{array}$ & $\begin{array}{l}\text { Puncture perpendicularly to a } \\
\text { depth of } 0.8-1.2 \text { cun }\end{array}$ \\
\hline & Zusanli (ST36) & $\begin{array}{l}3 \text { cun directly below Dubi (ST35), and one finger-breadth lateral to the an- } \\
\text { terior border of the tibia }\end{array}$ & $\begin{array}{l}\text { Puncture perpendicularly to a } \\
\text { depth of } 1.0-1.2 \text { cun }\end{array}$ \\
\hline & $\begin{array}{l}\text { Yanglingquan } \\
(G B 34)\end{array}$ & in the depression anterior and distal to the head of the fibula & $\begin{array}{l}\text { Puncture perpendicularly to a } \\
\text { depth of } 1.0-1.2 \text { cun }\end{array}$ \\
\hline & $\begin{array}{l}\text { Sanyinjiao } \\
\text { (SP6) }\end{array}$ & $\begin{array}{l}3 \text { cun superior to the prominence of the medial malleolus, posterior to the } \\
\text { medial border of the tibia, }\end{array}$ & $\begin{array}{l}\text { Puncture perpendicularly to a } \\
\text { depth of } 1.0-1.2 \text { cun }\end{array}$ \\
\hline & Taixi $(K / 3)$ & $\begin{array}{l}\text { in the depression between the prominence of the medial malleolus and } \\
\text { the calcaneal tendon }\end{array}$ & $\begin{array}{l}\text { Puncture perpendicularly to a } \\
\text { depth of } 0.5-0.8 \text { cun }\end{array}$ \\
\hline & $\begin{array}{l}\text { Bafeng }(E X- \\
\text { LE10) }\end{array}$ & $\begin{array}{l}\text { on the dorsum of the foot, between the first and fifth toes at the junction } \\
\text { of the red and white skin posterior to the margin of the web; } 4 \text { points on } \\
\text { each foot, } 8 \text { in total }\end{array}$ & $\begin{array}{l}\text { Puncture obliquely to a } \\
\text { depth of } 0.5-0.8 \text { cun }\end{array}$ \\
\hline $\begin{array}{l}\text { Additional acupoints for } \\
\text { upper limbs syndrome }\end{array}$ & Baxie (EX-UE9) & $\begin{array}{l}\text { at the dorsum of the hand, between the first and fifth fingers, proximal to } \\
\text { the web margins between the five fingers at the junction of the red and } \\
\text { white skin, } 4 \text { points on each hand, } 8 \text { in total }\end{array}$ & $\begin{array}{l}\text { Puncture obliquely upward } \\
\text { to a depth of } 0.5-0.8 \text { cun }\end{array}$ \\
\hline
\end{tabular}

${ }^{a} 1$ cun $(\approx 20 \mathrm{~mm})$ is defined as the width of the interphalangeal joint of patient's thumb 


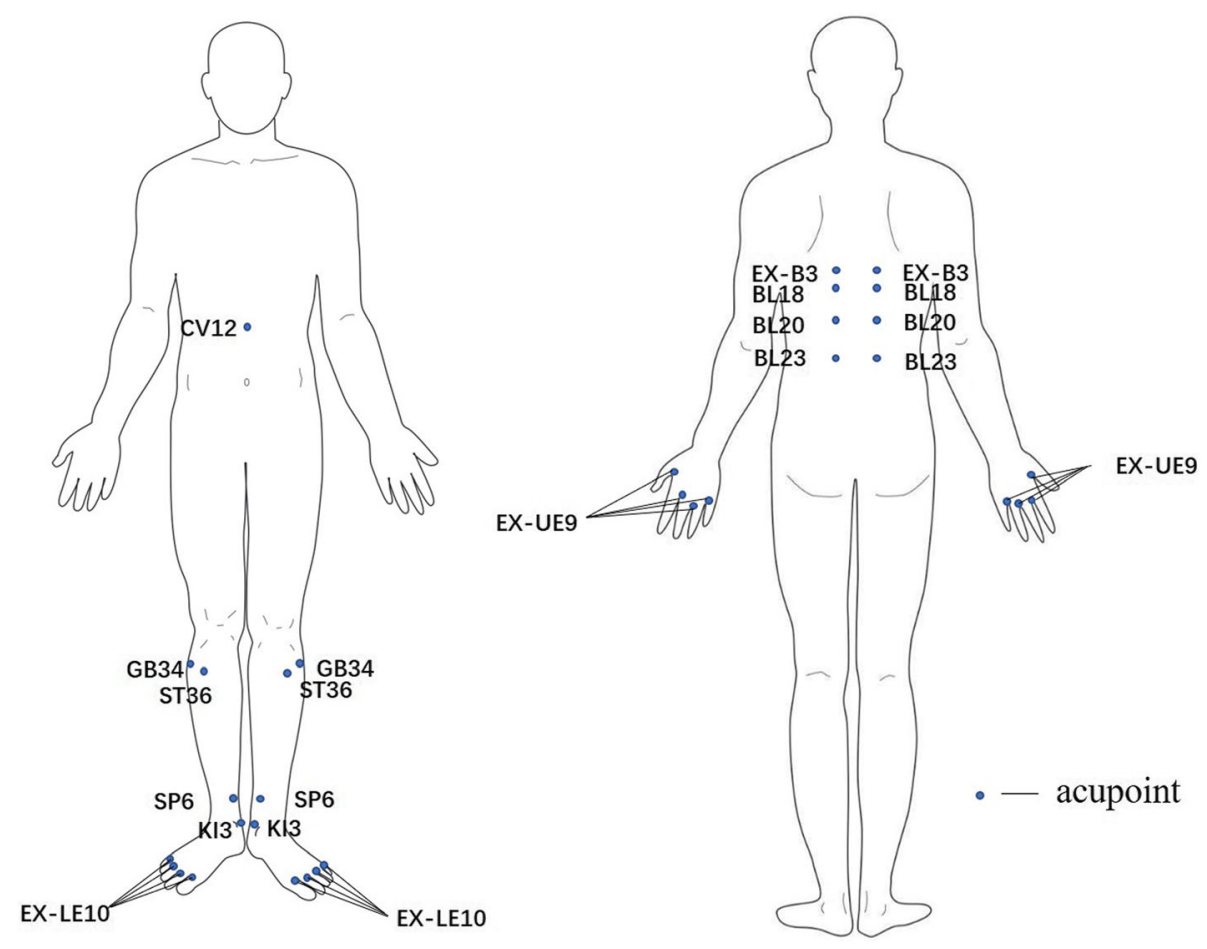

Fig. 2 Locations of acupoints

\section{Sham acupuncture}

Patients in the SA group will receive sham acupuncture. The procedure and duration of treatment in the SA group will be identical in the MA group except the needles $(0.25 \times 25 \mathrm{~mm}$, blunt tip, provided by Wuxi Jiajian Medical Instrument Co. Ltd., China) are blunt tip and there will be no skin penetration and needle manipulation for De qi.

Although it is difficult to set an eligible placebo control, using blunt-tipped needles that do not penetrate the patient's skin is the most common approach for administering sham treatments among acupuncture trials, according to a literature review [16, 17]. Moreover, this study will exclude those who have received acupuncture treatment in the last 3 months and can distinguish SA from MA. The same control with no skin penetration at acupoints was adopted and succeeded in masking patients with DPN $[7,14,15,18]$. Furthermore, all patients will be asked to guess which treatment they have received to test the patient-blinding effects at week 12 .

\section{Concurrent treatments}

Participants will receive routine health care as provided to all other patients with DPN. These treatments include glucose control, antihypertensive therapies, dyslipidemia control, analgesic, and neurotrophic treatments, if necessary. All information regarding the use of medications (including date of administration, types, and dosage) will be recorded. This will contribute to improving adherence.

\section{Follow-up}

After 12-week treatments, all participants will enter an additional 3 months follow-up period. During this time, they will receive routine health care as provided to all other patients with DPN. However, acupuncture treatment will not be permitted during follow-up.

\section{Outcomes}

Primary outcome

The primary outcome is peroneal motor nerve conduction velocity (peroneal $\mathrm{MNCV}$ ) which will be assessed at baseline and at week 12 after randomization.

\section{Key secondary outcomes}

Secondary outcomes include peroneal motor nerve action potential amplitude (peroneal MNAP) and latent period (peroneal MNLP), sural sensory nerve conduction velocity (sural SNCV), action potential amplitude (sural SNAP), and latent period (sural SNLP). The outcome measurements will be assessed at baseline and at week 12 after randomization.

Additional secondary outcomes include glycated hemoglobin (HbA1c), fasting plasma glucose (FPG), 2-h postprandial blood glucose (2hPG), body mass index (BMI), and 
blood pressure (BP). The outcome measurements will be assessed at baseline and at week 12 after randomization.

\section{Michigan Neuropathy Screening Instrument (MNSI) score}

The MNSI is a clinical and semi-quantitative evaluation of neuropathy that includes medical history and physical assessment. Medical history will be completed by patients with scores ranging between 0 and 13. Physical assessment will be completed by health professionals with five indicators and the aggregate score ranging between 0 and 10: foot appearance ( 0 and 1 for normal and abnormal, respectively), ulceration ( 0 and 1 for normal and abnormal, respectively), ankle reflex $(0,0.5$, and 1 for normal, reenforced, and absent, respectively), vibration test (0, 0.5, and 1 for normal, weakened, and absent, respectively), and monofilament examination $(0,0.5$, and 1 for normal, weakened, and absent, respectively) of feet on both sides. The outcome measurement will be assessed at baseline, at week 12 and at month 6 after randomization.

\section{Quality of life (QoL)}

Disease-specific QoL will be assessed at baseline and at week 12, month 6 after randomization using Diabetes Specific Quality of Life scale (DSQL). The scale consists of four domains: interference (12 items), psychology (8 items), social relations ( 4 items), and treatment (3 items). Each item is measured with a 5-point Likert scale ranging from "not at all" to "extremely". Higher scores indicate worse QoL.

\section{Patients' global assessment of DPN}

Patients' global assessment of DPN will be evaluated on a 5-point Likert scale at week 12. Patients will be asked to respond to the following question: "Considering all the ways your DPN affects you, how are you doing today?" 1 = very poor; 2 = poor; 3 = fair; 4 = good; or $5=$ very good.

\section{Blinding assessment}

To test the patient-blinding effects, all patients will be asked to guess their group assignment allocation within 2 min after the last treatment session at week 12 as following: "Which group do you think you are in?" A. traditional acupuncture; B. modified acupuncture; or C. not sure.

\section{Credibility and expectancy}

Credibility and expectancy of patients will be assessed by a Likert-scale Credibility/Expectancy Questionnaire (see Table 2) before the first treatment.

\section{Adverse events}

AEs data will document the occurrence, duration, and severity of adverse reactions (symptoms and signs), and how the event was resolved (or not) during the treatment. Based on their potential association with the acupuncture needling procedure, AEs will be categorized by acupuncturists and related specialists as treatmentrelated or not within $24 \mathrm{~h}$ after occurrence. Common treatment-related AEs include local subcutaneous hematoma, itching at the sites of needle insertion, continuous post-needling pain, dizziness, and so on. All participants will receive routine blood test, liver function (alanine transaminase and aspartate transaminase), and kidney function tests (serum creatinine and blood urea nitrogen). These tests will be performed twice, after randomization and at the end of 12-week treatment period. Serious adverse events will be reported to Medical Ethics Committee and the participant will be treated with relevant conventional therapy or hospitalization if necessary (the participant's allocated intervention will be revealed).

\section{Assessment of safety}

Patients will be asked to assess safety of treatments after 12 weeks' treatments, using a four-grade scale: safe, relatively safe, unsafe, and very unsafe.

\section{Sensation during the treatment}

Patients will be asked about their sensation during each treatment period as following: "What sensation do you feel during the treatment? A. soreness; B. distention; C. pain; D. no feeling; E. else, please specify

Table 2 Expectations for the efficacy of acupuncture are varied. If using following statements to describe your views on your disease/symptoms after the entire course of treatment, how much do you agree? For each statement, please choose the closest answer

\begin{tabular}{|c|c|c|c|c|c|}
\hline & Strongly disagree & Disagree & Neutral & Agree & Strongly agree \\
\hline a. My illness will get much better. & $\square_{1}$ & $\square_{2}$ & $\square_{3}$ & $\square_{4}$ & $\square_{5}$ \\
\hline b. I will face my disease better. & $\square_{1}$ & $\square_{2}$ & $\square_{3}$ & $\square_{4}$ & $\square_{5}$ \\
\hline c. Symptoms of my disease will disappear. & $\square_{1}$ & $\square_{2}$ & $\square_{3}$ & $\square_{4}$ & $\square_{5}$ \\
\hline d. I will be more vigorous than before. & $\square_{1}$ & $\square_{2}$ & $\square_{3}$ & $\square_{4}$ & $\square_{5}$ \\
\hline
\end{tabular}




\section{Usage of medication}

Medication Usage Log: Participants will be given a printed $\log$ to record their daily intake of prescribed medications. We will measure outcomes at week 12 .

The schedule of enrollment, interventions, and assessments are presented in Fig. 3.

\section{Data collection, management, and monitoring}

All researchers including acupuncturists, outcome assessors, and statisticians will receive training regarding data management. Case Report Forms (CRFs) will be completed and double entered into the Electronic Data Capture (EDC) system by two independent investigators to ensure accuracy of data.

All research documents, including both paper files and electronic documents, will be preserved for at least 5 years after publication. If reviewers or readers have any questions regarding our published data, they can contact the corresponding author for access to original data or visit ResMan (http://www.medresman.org/uc/

\begin{tabular}{|c|c|c|c|c|c|}
\hline \multirow[b]{3}{*}{ TIMEPOINT } & \multicolumn{5}{|c|}{ STUDY PERIOD } \\
\hline & \multirow{2}{*}{$\begin{array}{c}\text { Enrolment } \\
-1 \text { week } \\
\end{array}$} & \multirow{2}{*}{$\begin{array}{c}\text { Allocation } \\
0 \\
\end{array}$} & \multicolumn{2}{|c|}{ Treatment period } & \multirow{2}{*}{\begin{tabular}{|l}
$\begin{array}{l}\text { Follow-up } \\
\text { period }\end{array}$ \\
6 month \\
\end{tabular}} \\
\hline & & & 1-11 week & 12 week & \\
\hline \multicolumn{6}{|l|}{ ENROLMENT: } \\
\hline \multirow{4}{*}{$\begin{array}{l}\text { Eligibility screen } \\
\text { Informed consent } \\
\text { Allocation } \\
\text { Credibility and expectancy }\end{array}$} & $\mathrm{x}$ & & & & \\
\hline & $x$ & & & & \\
\hline & & $\mathrm{X}$ & & & \\
\hline & & $x$ & & & \\
\hline \multicolumn{6}{|l|}{ INTERVENTIONS: } \\
\hline \multirow{2}{*}{$\begin{array}{l}\text { Acupuncture group } \\
\text { Sham Acupuncture group }\end{array}$} & & & $\mathrm{x}$ & $\mathrm{x}$ & \\
\hline & & & $\mathrm{x}$ & $\mathrm{x}$ & \\
\hline \multicolumn{6}{|l|}{ ASSESSMENTS: } \\
\hline \multirow[t]{2}{*}{ peroneal MNCV MNAPI MNLP } & $\mathrm{x}$ & & & $\mathrm{x}$ & \\
\hline & $x$ & & & $\mathrm{x}$ & \\
\hline MNSI & $x$ & & & $x$ & $x$ \\
\hline DSQL & $\mathrm{x}$ & & & $\mathrm{x}$ & $\mathrm{x}$ \\
\hline HbA1c & $\mathrm{x}$ & & & $\mathrm{x}$ & \\
\hline FPG & $x$ & & & $\mathrm{x}$ & \\
\hline 2hPG & $\mathrm{x}$ & & & $\mathrm{X}$ & \\
\hline BMI & $\mathrm{x}$ & & & $\mathrm{x}$ & \\
\hline BP & $x$ & & & $\mathrm{x}$ & \\
\hline Routine blood test & $x$ & & & $\mathrm{x}$ & \\
\hline Liver function test & $x$ & & & $\mathrm{x}$ & \\
\hline Kidney function test & $x$ & & & $\mathrm{x}$ & \\
\hline Usage of medication & $\mathrm{x}$ & & & $\mathrm{x}$ & \\
\hline Sensation during the treatment & & & $\mathrm{x}$ & $\mathrm{x}$ & \\
\hline Adverse events & & & $\mathrm{x}$ & $\mathrm{x}$ & \\
\hline Blinding assessment & & & & $\mathrm{x}$ & \\
\hline Patients' global assessment & & & & $\mathrm{x}$ & \\
\hline Assessment of safety & & & & $x$ & \\
\hline
\end{tabular}

Fig. 3 The schedule of enrolment, interventions, and assessments 
project/projectadd.aspx). Private information of patients including name, telephone number, and ID number will be anonymous to ensure participant confidentiality.

Safety of this study will be monitored by a team consists of independent clinical experts and statisticians with access to unblinded data from Data and Safety Monitoring Board (DSMB) of the clinical evaluation center of Long-Hua Hospital, affiliated to Shanghai University of TCM. DSMB is independent from the sponsor, the competing interests, and the investigational site and will review the performance and safety of the trial every 6 months.

The criteria for unblinding and discontinuing allocated interventions for any trial participant experiencing serious acupuncture-related AEs have been described previously. DSMB will reveal a participant's allocated intervention and make the final decision on whether to terminate the trial.

\section{Statistical methods Sample size}

Based on results of a previous study, a sample size of 47 participants per group was estimated to provide $80 \%$ power to detect a between-group difference of $2.4 \mathrm{~m} / \mathrm{s}$ of conduction velocity improvement of common peroneal nerve, with a pooled standard deviation of $4.15 \mathrm{~m} / \mathrm{s}$ and an alpha level of 0.05 . The assumption of a conservative $20 \%$ drop-out rate in any group increases the sample size to 59 participants per group or 118 participants in total.

\section{Statistical analysis}

Statistical analysis will be performed by an independent statistician who is not aware of group allocation. SPSS 21.0 (IBM SPSS Statistics, New York, USA) will be used for data analysis. Level of significance will be established at $\alpha<0.05$ with a two-sided test. Continuous data will be represented by mean \pm standard deviation or median (range), and categorical data will be represented by percentage. All efficacy analyses will be performed using the intent-to-treat (ITT) approach. For the ITT analysis, population will consist of all patients who have been randomized. Missing data will be imputed using the last observation carried forward (LOCF) method. Continuous variables will be compared using Student's $t$ test or Wilcoxon rank-sum test as appropriate. Categorical variables will be compared using Fisher's exact test or Wilcoxon rank-sum test as appropriate. Analysis of covariance (ANCOVA) or logistic regression will be used for analysis and adjustment of baseline characteristics that differ significantly between two groups.

\section{Ethics and dissemination}

The study protocol which follows principles of the Declaration of Helsinki has been approved by the Medical
Ethics Committee of Long-Hua Hospital affiliated to Shanghai University of Traditional Chinese Medicine since 7 July 2016 (Approval Number: 2016LCSY028) and registered on the Chinese Clinical Trial Registry, ChiCTR1800020444. Results will be disseminated through peer-reviewed publications, a master's thesis, or conference presentations. Data will be anonymized prior to publication to prevent identification of individual participants.

\section{Discussion}

Diabetes mellitus (DM) is a common disease which is accompanied by highly significant social and economic burdens [19]. According to the International Diabetes Federation (IDF), DM affects 415 million people worldwide, with a prevalence of $8.8 \%$, and these values are expected to rise still further to 642 million by the year 2040 , with a prevalence of $10.4 \%$ [20]. DPN is one of the most frequent complications of DM and usually characterized by gradually increases in pain severity, impairments in tactile and proprioceptive sensation, vibration sense, and improper postural control [21]. DPN is commonly associated with high rates of mortality and morbidity [22]. Acupuncture has been widely used in clinical practice for the treatment of DPN in China. However, so far there has been no appropriately designed randomized controlled trial to provide clear evidence about the effectiveness of acupuncture treatment for DPN at home and abroad.

In this study, the inclusion criterion of age, from 18 to 75 years, was chosen to cover age range as wide as possible. We cooperated with the endocrinology department of Long-Hua Hospital affiliated to Shanghai University of Traditional Chinese Medicine to ensure the required number of subjects recruited. During the study period, patients will receive routine health care as provided to all other patients with DPN. Usually, we encourage them not to change their medications, but adjusting medications will be permitted if necessary. We believe this strategy can reflect real-world practice and satisfy ethical obligations better.

A standardized treatment protocol will be utilized to assure reproducibility of the study. In this trial, the treatment protocol was based on traditional acupuncture theory, previous studies [15], and the consensus of endocrinologists and acupuncturists from Long-Hua Hospital affiliated to Shanghai University of Traditional Chinese Medicine. The amount of acupuncture is intensive, which is similar to clinical practice in China. Needles will be stimulated manually for at least $10 \mathrm{~s}$ at each acupoint and retained in place for $20 \mathrm{~min}$. There will be 2 treatment sessions per week within the course of 12week treatment, giving a total of 24 sessions.

A suitable control group is critical for a well-designed clinical trial. On the basis of literature review, using a 
blunt-tipped needle that does not penetrate the patient's skin is the most commonly used approach for administering sham treatments among acupuncture trials $[16,17]$. Patients who have received acupuncture treatment in the last 3 months and can distinguish SA from MA will be excluded. The same control group design, no skin penetration at acupoints, was adopted and succeeded in masking patients with DPN $[7,14,15,18]$. Furthermore, all patients will be asked to guess which treatment they have received to test patient-blinding effects at week 12 .

The change of nerve conduction velocity is chosen as the primary outcome because it is the highest sensitive and objective method to detect neuropathy. Multiple systematic reviews [11] recommended nerve conduction velocity as a main outcome, and it had been widely used as an outcome measure in DPN studies [23]. At the same time, assessment of the severity of DPN mainly depends on patient-reported outcomes. The MNSI is the most DPN-specific patient-reported measure. In this trial, we use the DSQL to evaluate quality of life in patients with DPN before and after acupuncture treatment. The DSQL captures the impact of detailed aspects of modern diabetes management (e.g., carbohydrate counting and flexible insulin dose adjustment) that is extensively used in evaluating quality of life in DM. The DSQL offers a valuable tool for assessing the impact of treatment approaches on QoL in adults with DM [20].

The limitation of our trial is acupuncturists are not blinded for the nature of intervention. We hope results of this trial will provide more reliable evidence and clarify the value of acupuncture as a treatment for DPN.

To summarize, this trial meets the methodological demand of adequate randomization and allocation concealment, blinding of patients, outcome assessors, and statisticians. Findings of this study will provide high-quality evidences for evaluating the efficacy and safety of acupuncture treatment for DPN.

\section{Trial status}

This trial is currently recruiting patients. This is version 2.0 of the protocol, dated June 6, 2016. Recruitment began on 10 October 2016 and the anticipated end date is 31 December 2021.

\section{Supplementary information}

Supplementary information accompanies this paper at https://doi.org/10. 1186/s13063-020-04811-3.

Additional file 1. Completed Standard Protocol Items: Recommendation for Interventional Trials (SPIRIT) 2013 Checklist: items addressed in this clinical trial protocol.

Additional file 2. Informed consent.

\section{Abbreviations}

ADA: The American Diabetes Association; AEs: Adverse events; BMl: Body mass index; BP: Blood pressure; CRF: Case report form; DM: Diabetes mellitus;
DPN: Diabetic peripheral neuropathy; DSMB: Data and Safety Monitoring Board; DSQL: Diabetes Specific Quality of Life scale; FPG: Fasting plasma glucose; HbAlc: Glycated hemoglobin; 2hPG: 2-h postprandial blood glucose; IDF: International Diabetes Federation; ITT: Intent-to-treat; MA: Manual acupuncture; MNAP: Motor nerve action potential amplitude; MNCV: Motor nerve conduction velocity; MNLP: Motor nerve action potential latent period; MNSI: Michigan Neuropathy Screening Instrument; PN: Peripheral neuropathy; QoL: Quality of life; SA: Sham acupuncture; SNAP: Sensory nerve action potential amplitude; SNCV: Sensory nerve conduction velocity;

SNLP: Sensory nerve action potential latent period

\section{Acknowledgements}

Zhiyi Qian for improvements to the English language.

\section{Authors' contributions}

$H P D, X Y S, H Y, H T$, and JP participated in the conception and design of this trial. $H P D, L Z, Y S$, and JP were responsible for planning the draft and revising the manuscript. JP and XYS are monitors of this study. KC is responsible for randomization and allocation concealment. HY and $\mathrm{HT}$ are responsible for the recruitment of patients and obtain informed consent. HPD, TTZ, and YS are responsible for the treatment of patients. YS and PRL are responsible for collecting the data. All authors have read the manuscript and approved the publication of this protocol

\section{Funding}

This study was supported by the Three-Year development plan project for Traditional Chinese Medicine of Shanghai Municipal Health Commission (grant numbers ZY (2018-2020)-CCCX-1006. The sponsor plays no role in study design, collection, management, analysis, interpretation of data, writing of the report, or the decision to submit the report for publication.

\section{Availability of data and materials}

All de-identified data collected during the trial will be available for anyone who wishes to access 6 months after publication in accordance with FAIR principles.

\section{Ethics approval and consent to participate}

The study is approved by the Medical Ethics Committee of Long-Hua Hospital affiliated to Shanghai University of Traditional Chinese Medicine, (Approval No. 2016LCSY028) and registered with Chinese Clinical Trial Registry, ChiCTR1800020444. Written informed consent will be obtained from all patients prior to their enrollment in the study.

\section{Competing interests}

The authors declare that they have no competing interests.

\section{Author details}

${ }^{1}$ School of Acupuncture-Moxibustion and Tuina, Shanghai University of Traditional Chinese Medicine, Pudong District, Shanghai, China. ²Shanghai Research Center of Acupuncture and Meridian, Pudong District, Shanghai, China. ${ }^{3}$ Long-Hua Hospital affiliated to Shanghai University of Traditional Chinese Medicine, Xuhui District, Shanghai, China.

Received: 13 April 2020 Accepted: 16 October 2020

Published online: 26 October 2020

\section{References}

1. Iqbal Z, Azmi S, Yadav R, Ferdousi M, Kumar M, Cuthbertson DJ, Lim J, Malik RA, Alam U. Diabetic peripheral neuropathy: epidemiology, diagnosis, and pharmacotherapy. Clin Ther. 2018;40:828-49.

2. Denise SH. Diabetic peripheral neuropathy: evaluation and management. J Nurse Pract. 2017:13:199-204

3. Gordon S, Pallai S, Dinesh S, Jing W, lain DW, Irene T, Praveen A, Solomon T. A new look at painful diabetic neuropathy. Diabetes Res Clin Pract. 2018; 144:177-91.

4. Won JC, Kim SS, Ko KS, Cha BY. Current status of diabetic peripheral neuropathy in Korea: report of a hospital-based study of type 2 diabetic patients in Korea by the Diabetic Neuropathy Study Group of the Korean Diabetes Association. Diabetes Metab J. 2014;38:2531. 
5. Ziegler D, Fonseca V. From guideline to patient: a review of recent recommendations for pharmacotherapy of painful diabetic neuropathy. J Diabetes Complicat. 2015;29:146-56.

6. Baute V, Zelnik D, Curtis J, Sadeghifar F. Complementary and alternative medicine for painful peripheral neuropathy. Curr Treat Options Neurol. 2019;21:44.

7. Garrow AP, Xing M, Vere J, Verrall B, Wang L, Jude E. Role of acupuncture in the management of diabetic painful neuropathy (DPN): a pilot RCT. Acupunct Med. 2014;32:242-9.

8. Anne B, Deborah W, Matthew A, Priscilla S, Daniel C. Acupuncture treatment of diabetic peripheral neuropathy in an American Indian community. J Acupunct Meridian Stud. 2017;10:90-5.

9. Franco LC, Souza LAF, Da Costa Pessoa AP, Pereira LV. Nonpharmacologic therapies in diabetic neuropathic pain: a review. Acta Paulista de Enfermagem. 2011;24(2):284-8.

10. Franconi G, Manni L, Schröder S, Marcetti P, Robinson N. A systematic review of experimental and clinical acupuncture in chemotherapy-induced peripheral neuropathy. Evid Based Complement Alternat Med. 2013. https:// doi.org/10.1155/2013/516916.

11. Dimitrova A, Murchison C, Oken B. Acupuncture for the treatment of peripheral neuropathy: a systematic review and meta-analysis. J Altern Complement Med. 2017;23:164-79.

12. Boulton AJM, Vinik Al, Arezzo JC, Bril V, Feldman EL, Freeman R, Malik RA, Maser RE, Sosenko JM, Ziegler D. Diabetic neuropathies: a statement by the American diabetes association. J Diabetes Care. 2005;28:956-62.

13. American Diabetes Association. Diagnosis and classification of diabetes mellitus. Diabetes Care. 2012;35(Suppl 1):S64-71.

14. Liu $B, X u H, M a R, M o ~ Q$, Yan $S$, Liu Z. Effect of blinding with a new pragmatic placebo needle: a randomized controlled crossover study. Medicine (Baltimore). 2014;93:e200.

15. Wang $Q$, Deng $H P$, Cheng $K$, Huang ZQ, Yin $X Q$, Zhou $Y C$, Yang $Y Q$, Shen WD, Zhao L, Shen XY. Manual acupuncture for the infertile female with polycystic ovary syndrome (PCOS): study protocol for a randomized shamcontrolled trial. J Trials. 2019;20:564.

16. Wang YR, Zhao JP, Hao DF. Is sham acupuncture a real placebo: skeptical for sham acupuncture. World J Acupunct Moxibustion. 2017:27:1-5.

17. Zhang LL, Chu Q, Wang S, Lai H, Xie BB. Is sham acupuncture as effective as traditional Chinese acupuncture? It's too early to say. Chin J Integr Med. 2016;22:483-9

18. Chan K, Lui L, Yu K, Lau K, Lai M, Lau W, Ng B, Zhong LLD, Bian ZX. Trials. 2020;21(1):58. https://doi.org/10.1186/s13063-019-3972-5.

19. Maronesi CT, Cecagno-Zanini SC, de Oliveira LZ, Bavaresco SS, Leguisamo CP. Physical exercise in patients with diabetic neuropathy: systematic review and meta-analysis of randomized clinical trials. J Fisioter Pesqui. 2016;23:216-23.

20. Cooke D, O'hara MC, Beinart N, Heller S, La Maeca R, Byrne M, Mansell P, Dinneen SF, Clark M, Bond R, Speight J. Linguistic and psychometric validation of the diabetes-specific quality-of-life scale in U.K. English for adults with type 1 diabetes. J Diabetes Care. 2013;36:1117-25.

21. Akbari M, Jafari H, Moshashaee A, Forugh B. Do diabetic neuropathy patients benefit from balance training? J Rehabil Res Dev. 2012;49:333-8.

22. Yamany AA, Sayed HM. Effect of low level laser therapy on neurovascular function of diabetic peripheral neuropathy. J Adv Res. 2012;3:21-8.

23. Tong Y, Guo H, Han B. Fifteen-day acupuncture treatment relieves diabetic peripheral neuropathy. J Acupunct Meridian Stud. 2010;3:95-103.

\section{Publisher's Note}

Springer Nature remains neutral with regard to jurisdictional claims in published maps and institutional affiliations.

Ready to submit your research? Choose BMC and benefit from:

- fast, convenient online submission

- thorough peer review by experienced researchers in your field

- rapid publication on acceptance

- support for research data, including large and complex data types

- gold Open Access which fosters wider collaboration and increased citations

- maximum visibility for your research: over $100 \mathrm{M}$ website views per year

At BMC, research is always in progress.

Learn more biomedcentral.com/submissions 